\section{Computer signal detection by monitoring auditory evoked potentials}

\author{
JAY W. BAUER, KENNETH C. SQUIRES, and PETER H. LINDSAY * \\ University of California, San Diego, La Jolla, California 92037
}

A digital computer, using a simple decision algorithm, attempted to determine when an acoustical signal had been presented to a cat by monitoring the amplitude of the evoked potentials (EP) at brainstem auditory nuclei. The signal-to-noise level at threshold and the shape and range of the decision model "psychometric functions" were similar to those obtained from humans in the same task. In addition, the detection performance obeys Weber's law, the mean amplitude of the EP increases monotonically with signal level, the variance of the amplitude is independent of both signal and noise level, and both the mean latency and the variance of the latency of the peaks decrease with increasing signal level. These findings suggest that the synchronization in firing of a population of single units plays a part in determining the amplitude of the EP. Interaural effects in detection performance were found at the inferior colliculus and, to a lesser extent, at the superior olive.

In these experiments, we used a computer program to model the decision component of an $O$ operating in a standard yes-no detection task. On a trial-by-trial basis, responses recorded from permanently implanted macroelectrodes in the auditory system of a cat were used as the input to the decision model. The computer monitored the response produced on a trial, computed decision statistics, and made a decision as to whether or not a signal had occurred on that trial. The performance characteristics of the decision model, together with the statistical properties of the neural responses, were studied under a variety of stimulus conditions commonly used in psychophysical studies.

Recently, there have been several attempts to determine which features of sensory neural activity could plausibly be the basis for the internal decision statistic which is explicitly assumed in signal detection theory. Hillyard, Squires, Bauer, and Lindsay (1971) demonstrated correlations in human detection performance with certain parameters in the average auditory evoked potential recorded from scalp electrodes. Taub and Raab (1969) computed "physiological difference limens" from observations of peak-to-peak amplitudes of round window potentials evoked by click signals presented to anesthetized guinea pigs and found that Weber fractions based on the physiological responses compared favorably with those for human Ss in the same task.

*This research was supported by NIH Grant NS 07454. We are indebted to Steve Sitko for his assistance in the implantation operation. We thank Drs. Robert Galambos, David Green, and Donald Norman for use of their various facilities and for commenting on earlier drafts of this paper.
Other features of the neural activity which can be related to external stimuli are the responses of single units, of multiple units, and average evoked responses from implanted electrodes. The response of a single unit is highly variable, whereas poststimulus time histograms of single-unit responses, or better, multiple-unit responses, are essentially invariant for a particular stimulus and are highly correlated with the average evoked potential. These findings of John and Morgades (1969) led them to suggest that in an unanesthetized animal it is the average response pattern of an extensive neural ensemble which is used to identify chose to investigate those features of single-trial evoked potentials (EP) in external stimuli. For this reason, we on data at three or four different noise levels. auditory brainstem nuclei which might be the basis for the hypothesized internal decision statistic of signal detection theory. It was felt that if a plausible neural statistic and decision-making algorithm consistent within the framework of signal detection theory could reliably predict observed detection behavior, a methodology would be established for determining how information from external stimuli is processed in the auditory system.

\section{PROCEDURE}

Two cats were used in the experiment: a 1-year-old female especially prepared for this study (FY) and a 4-year-old male (MO) whose electrodes had been implanted approximately 3 years prior to this study. ${ }^{1}$ Electrodes were implanted using a stereotaxic instrument and a standard operating procedure. The electrodes were Teflon-coated bipolar stainless steel with tip diameters of approximately 200 microns. Both cats had a bilateral implant in the inferior colliculus and a unilateral implant in the superior olive and the cochlear nucleus. The electrodes were terminated in a subminiature connector which was permanently fixed to the skull. For the cat FY, data recording began 1 month after the operation.

Recordings were made with the cat unanesthetized and lightly restrained in a box inside a double-walled acoustic chamber. Recordings were monopolar referenced to a skull screw and band-passed between $8 \mathrm{~Hz}$ and $1 \mathrm{kHz}$. The electrode responses were amplified 10,000 times by Tektronix Type 122 amplifiers and sampled by the analog-to-digital converter on a

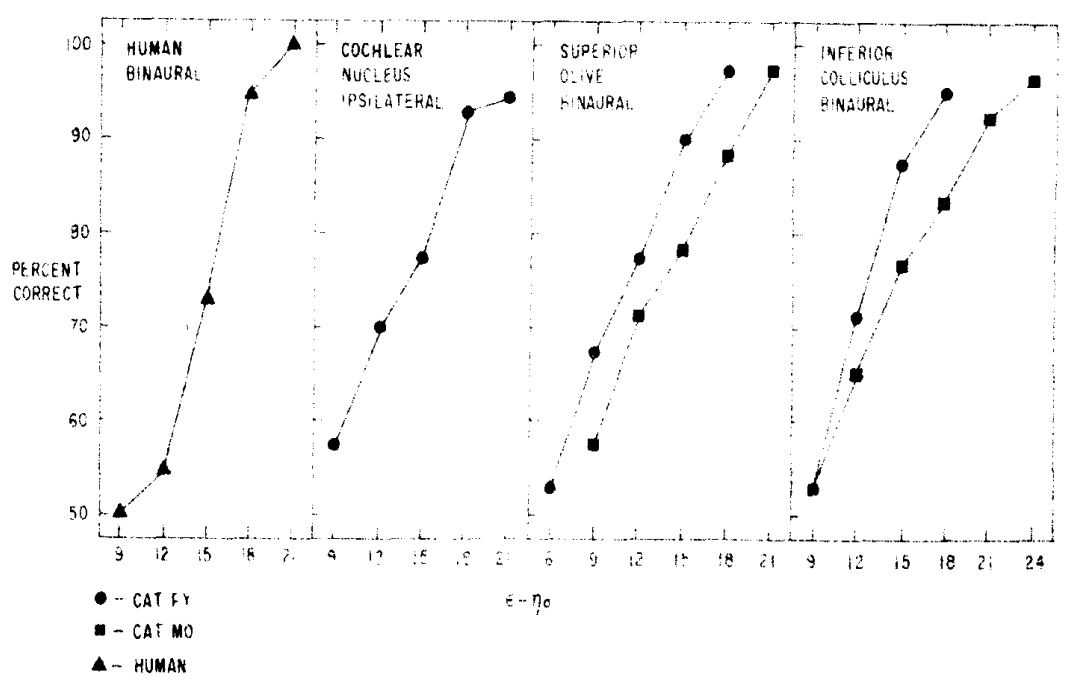

Fig. 1. Psychometric functions from both the human $O$ and the computer decision model. Each point is based on 300 or 400 trials. Each function is based 


\begin{tabular}{|c|c|c|c|c|c|}
\hline \multirow[b]{2}{*}{$\epsilon-\eta_{0}$} & \multirow[b]{2}{*}{$\begin{array}{l}\text { PERCENT } \\
\text { CORRECT }\end{array}$} & \multicolumn{2}{|c|}{$\begin{array}{l}\text { SUPERIOR OLIVE } \\
\text { BINAURAL }\end{array}$} & \multicolumn{2}{|c|}{ CAT FY } \\
\hline & & HITS & MISSES & $\begin{array}{l}\text { FALSE } \\
\text { ALARMS }\end{array}$ & $\begin{array}{l}\text { CORRECT } \\
\text { REJECYIONS }\end{array}$ \\
\hline
\end{tabular}

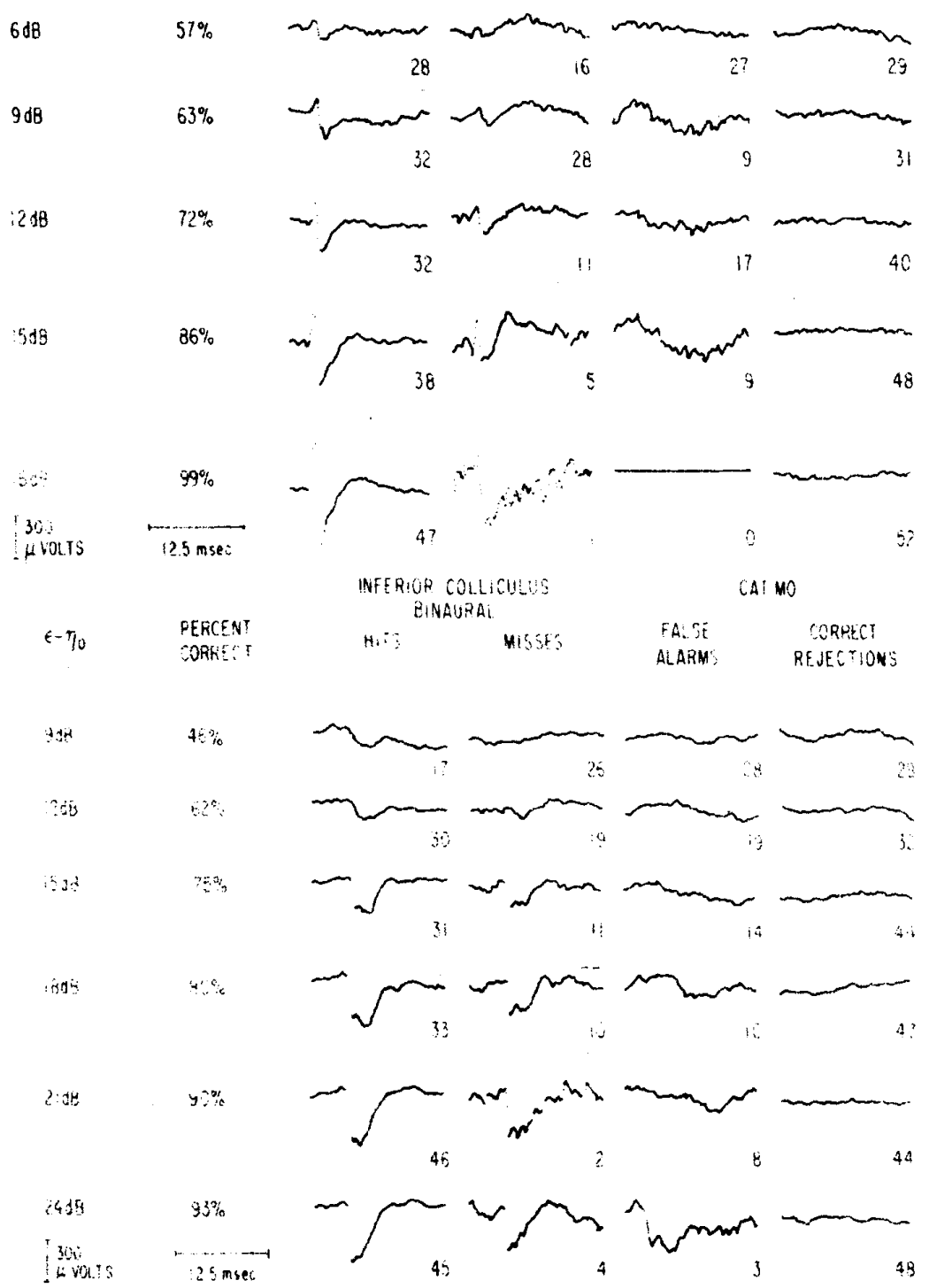

Fig. 2. Averaged EPs collected according to the four different stimulus-response conditions (hit, miss, false alarm, and correct rejection) as a function of $\epsilon-\eta_{0}$ (dB). The corresponding percent correct is given for each signal-to-noise ratio. The number of trials upon which each averaged wa veform is based is presented below the waveform. Both the inferior collicular and the superior olivary EPs were obtained at a $\eta_{0}$ level of $11 \mathrm{~dB}$.

PDP-9 computer to 12-bit accuracy at a sampling rate of $20 \mathrm{kHz}$. The evoked response from only one center was monitored during each block of trials.

The task was to detect the occurrence of the signal-a click produced by a single cycle of a $5-\mathrm{kHz}$ square wave gated on at the positive-going zero crossing-which occurred on any trial with probability 0.5. There was a background of continuous white noise. In the binaural condition, the click signal and background noise were presented in phase. Acoustic signals were delivered to the cat through Audivox 9C miniature earphones mounted on the skull connector and positioned inside the pinna at the entrance to the external auditory meatus. The click voltage-controlled generator. The signal and noise parameters remained constant within each block of 100 signals were produced by a Wavtek trials. Trials were presented once every 2 sec, with the computer controlling the timing, randomization, and delivery of the signal, as well as the data recording and decision analysis. The decision-making part of the program did not know what signal had been presented; it made its decision only on the received EP input.

$A$ human $\mathrm{O}$ also took part in the same sequence of experiments, the only difference being that signals were presented to him over Grason-Stadler TDH-39 earphones with supraaural earpads. No physiological measurements were made. Feedback was not given.

The sound pressure levels for the Grason-Stadler TDH-39 earphones were determined using a 5.642-cc coupler (Type GR 9A) and a General Radio sound level meter, Type 1561-A. The Audiovox 9-C earphones were calibrated using the same equipment modified for use with the Audiovox earphones. The modification did not alter the volume of the coupler. ${ }^{2}$

\section{The Decision Strategy}

The EP of the auditory center being investigated was sampled for a 12.5-msec period synchronized to the start of a trial. The first stage in the decision process was to compute the maximum peak-to-peak amplitude deflection, the latencies of and the areas under the positive and negative peaks. These amplitude and area statistics were then passed on to the second stage of the program which used them to decide whether or not a signal had occurred on that trial. ${ }^{3}$

The decision program decided whether or not a signal had occurred by comparing the current trial amplitude value against a criterion established during the preceding trials. of the many decision rules possible, we have used only one: the decision program reported "signal" whenever the value of the current trial statistic exceeded the average value computed from all preceding trials and "no signal" whenever the computed trial value was at or below this average. When signals are presented with probability 0.5 , this decision rule maximizes the overall percent correct performance (hits plus correct rejections) and produces symmetrical error rates (misses and false alarms), 4 given that the underlying sensory distributions are symmetrical.

A parameter of the decision program controlled the amount of evidence accumulated before actually making decisions. Obviously, with this decision rule, on any new block of trials the program starts with no information and can only make guesses while the criterion is 
stabilizing. Thus, practice trials were used-five presentations each of both stimuli. Practice trials were not included in tabulating overall detection performance, but they were included in determining the characteristics of the decision statistic over a block of trials.

After making the decision, control passed to the analysis portion of the program which tabulated decision performance according to the particular combination of signal-response events that occurred on the trial (i.e., the number of hits, false alarms, misses, and correct rejections), as well as recording the values and latencies of the various components of the waveform. Finally, the distributions of the decision statistics and the average EPs produced under the different signal-response combinations were accumulated across trials.

\section{RESULTS AND DISCUSSION}

Physiological Thresholds

Figure 1 shows "psychometric functions" based on the performance of the decision model monitoring several different nuclei in the auditory system of the two cats and the psychometric function for the one human $O$ detecting the same signal in broadband white noise in a yes-no detection task. 5 Figure 2 shows how the average EP for each of the four different signal-response conditions (hits, misses, false alarms, correct rejections) varies as a function of the signal-to-noise ratio. The average EPs on the false alarm and correct rejection trials appear flat because they are averaged samples of random noise, not synchronized activity. In any single EP, however, the peak-to-peak amplitude in response to noise alone might actually have measured 100 or 200 microV. The decision model given such a statistic would incorrectly respond that a signal had been presented, thereby generating a false alarm. At high signal-to-noise ratios, a signal presentation usually evokes a very large response, which temporarily may produce a relatively high criterion value, especially in the early trials of a block. As a result, moderately large EPs are sometimes not detected by the decision model, as seen in the average evoked response for misses at high signal levels in Fig. 2.

Overall, the performance of a decision model using evoked responses from brainstem nuclei as inputs appears remarkably similar to the behavior of human $\mathrm{Os}$ in the same task. The general ogival shape and the range of the psychometric functions are similar, though less steep for the decision model. ${ }^{6}$ Detection thresholds

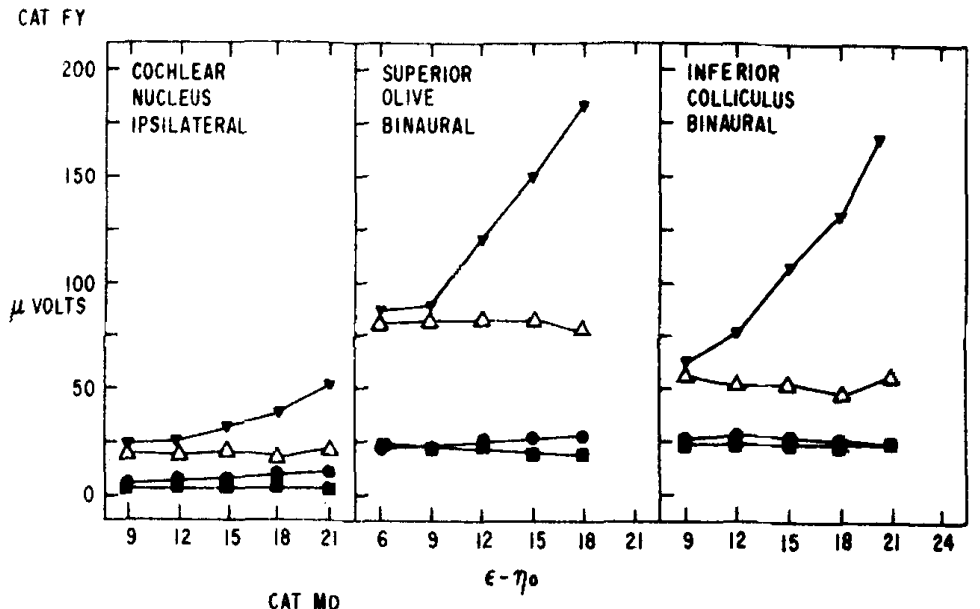

CAT MO

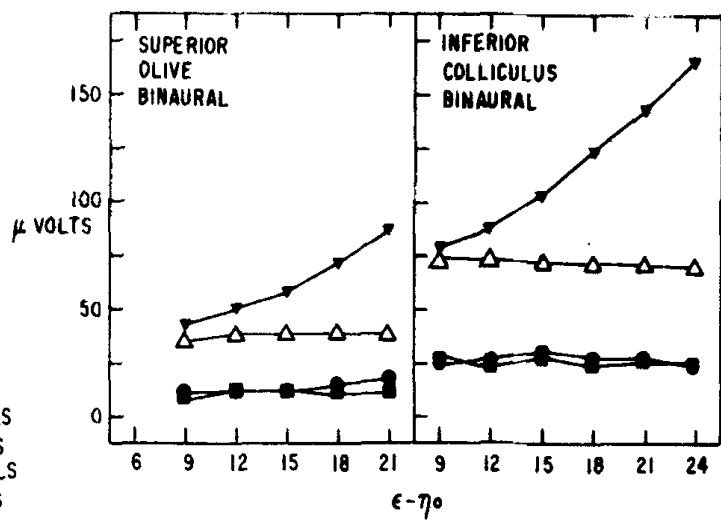

Fig. 3. Peak-to-peak amplitude statistics (mean and standard deviation) in microvolts for both signal and noise trials as a function of $\epsilon-\eta_{0}$ (dB). Each data point is based on approximately 150 trials.

based on the amplitude of the physiological response resemble those found in our human detection experiment: signal-to-noise ratios for $75 \%$ correct detection by the decision model ranged from 10 to $18 \mathrm{~dB}$ at different levels in the auditory system under several different listening conditions, as opposed to approximately $15 \mathrm{~dB}$ for binaural thresholds in our human detection task. For human detection tasks involving pure tone signals, signal-to-noise ratios at $75 \%$ correct detection range from 6 to $15 \mathrm{~dB}$ over a frequency range of 250 to $4,000 \mathrm{~Hz}$ (Green, McKey, \& Licklider, 1959). Thus, detection performance, based directly on the amplitude of the EP in the cat brainstem, would seem to be reasonably similar to observed behavioral measures in humans. This is reassuring since if there were no correspondence between detection performance of the decision model and human performance, the validity of this approach would be immediately suspect.

The Statistics of

Neural Responses

For theorizing about the neural coding of auditory information, it is important to separate those conditions which alter the variability in the underlying neural responses from those which change the average value of the neural statistic used to make decisions. Figure 3 shows the mean values and standard deviations of the amplitude statistic as a function of signal-to-noise ratio for both signal trials and noise trials. The values for noise trials are simply the average of the maximum noise-generated neural deflections found with regular samples of the ongoing background activity. Since the external noise level was constant as the signal-to-noise ratio was varied over the range from chance detection levels to $100 \%$ correct detection levels, we would expect both the mean and standard deviation of the response amplitudes on noise trials to be independent of the extermal signal-to-noise ratio, and this can be seen in the figure.

However, the variability in the amplitude statietic on signal trials is also unaffected by increaning the signal level over the range of intensities spanned by the psychometric function of the decision model. Moreover, the 


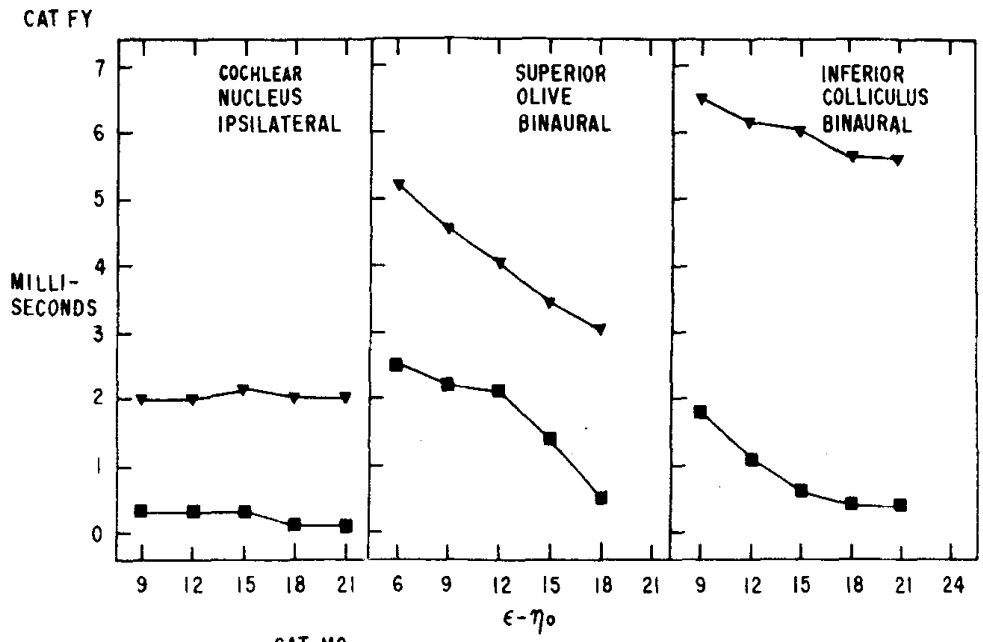

CAT MO

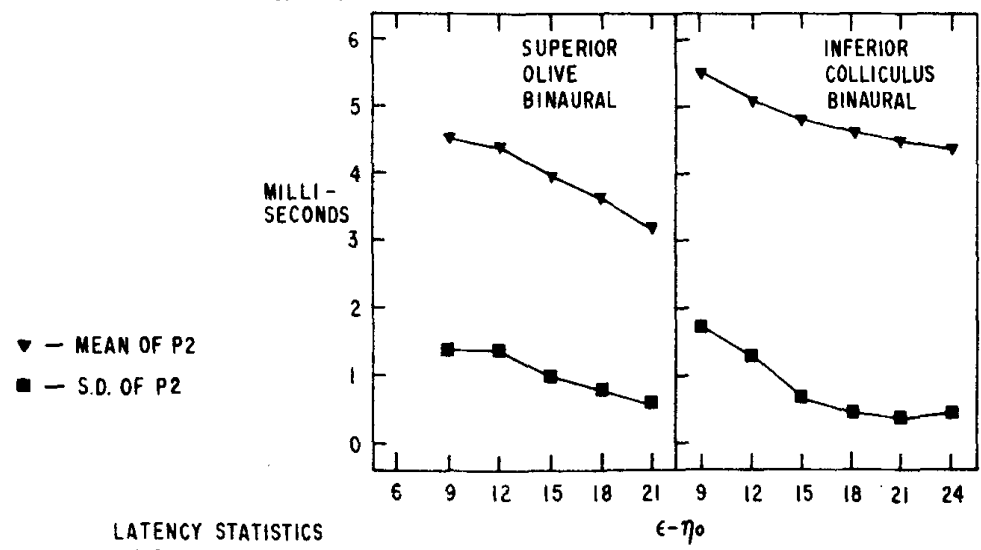

ON SIGNAL. TRIALS

Fig. 4. The mean and standard deviation of the latency in milliseconds of the occurrence of the first positive peak on signal trials alone as a function of $\epsilon-\eta_{0}$ (dB). Each point is based on approximately 150 trials.

standard deviation is approximately the same as that of ongoing neural activity in the presence of continuous noise. Improvement in detection performance at a given noise level, then, appears to be accounted for exclusively by a systematic increase in the separation of the sensory distributions associated with increasing the signal-to-noise ratio.

However, the change in the amplitude measure as the signal-to-noise ratio increases is not the only change in the EP. Figure 4 shows the changes in the temporal characteristics of the waveform as the signal-to-noise ratio is increased. The latency of the maximum amplitude systematically decreases with increased signal-to-noise ratios. More importantly, the trial-to-trial variation in when the peak occurs also decreases systematically. This decrease in the variability of the latency response would be expected if the increased signal-to-noise ratio was increasing the synchronization in the flow of nerve impulses. .distributions plotted on normal probability paper. These distributions are statistically nonnormal (Pearson goodness-of-fit chi-square tests for normality of a distribution demonstrate that these distributions are not normal at a significance level beyond 0.001 ). However, Fig. 6 shows that the sensory distributions approximate normality over a large extent of their range. Several of the distributions are skewed in at least one direction. This skewness and the large sample size probably account for the highly significant chi-square tests. It should be noted that such skewness has been proposed to account for the asymmetry in the ROC curves of human Os (see Green \& Swets, pp. 78-81, 1966).

\section{Weber's Law}

Figure 7 shows psychometric functions for the decision model at different external noise levels. In Fig. 8 , we have calculated the signal energy required for $75 \%$ correct detection at varying noise levels from the decision model psychometric functions for all three brainstem nuclei and from the human psychometric functions. The straight lines are of unit slope: the performance expected according to Weber's law, where the signal energy required to achieve a constant detection performance is proportional to the background noise level. Within the range of signal conditions investigated, Weber's law appears to provide an excellent approximation to changes in detectability obtained from the decision model as a function of external noise level for all three brainstem nuclei in the cat.

The performance characterized as Weber's law may arise in any detection system in which the standard deviation (i.e., "error") of the process making the measurements is proportional to the quantity being measured. This assumption about "internal noise" is the usual explanation of Weber's law when it is found in human detection and discrimination tasks (see Green \& Swets, 1966, pp. 226-229). When external background noise is present, increasing the noise level is assumed to increase the variability in the sensory response. In addition, the amount of "internal noise" added during the processing of a signal may be proportional to the signal level. Figure 9, however, shows that the standard deviation of the amplitude statistic on signal trials and on noise trials for signal levels producing $75 \%$ correct detection is not increasing with signal or noise level.

That the variability of the EP amplitude is independent of both the noise and signal level does not agree 


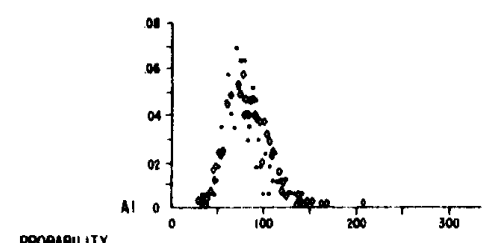

PROARELIIY

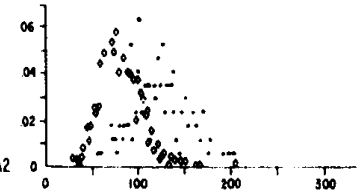

DENSITY

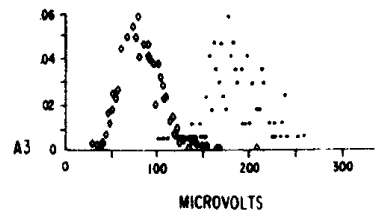

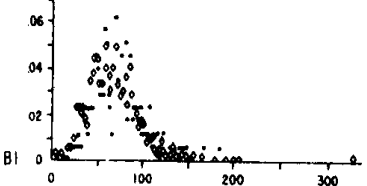

PROBABILITY

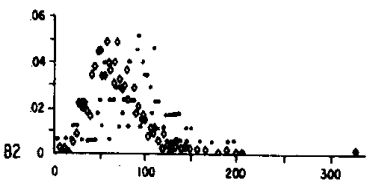

OENSITY

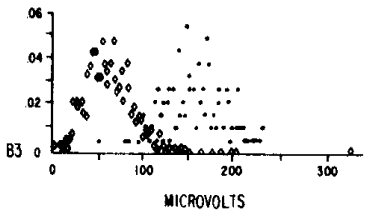

Fig. 5. Distributions of individual EP peak-to-peak amplitudes in microvolts at different levels of $\epsilon-\eta_{0}(\mathrm{~dB})$ and of percent correct: (A1) $6 \mathrm{~dB}$ and $53 \%$, (A2) $12 \mathrm{~dB}$ and $78 \%$, (A3) $18 \mathrm{~dB}$ and $98 \%$, (B1) $9 \mathrm{~dB}$ and $53 \%$, (B2) $15 \mathrm{~dB}$ and $76 \%$, and (B3) $24 \mathrm{~dB}$ and $97 \%$. The distributions have been scaled so that the sum of the points for the signal distributions and noise distributions are both approximately equal to one. Noise distributions are based on 750 to 900 trials, whereas signal distributions are based on 150 to 200 trials.

with the findings of Frishkopf (1956) and Taub and Raab (1969), who, recording differentially from the round window of cats and of guinea pigs, respectively, found that the standard deviation of $\mathrm{N}_{1}$ first increased and then decreased as signal intensity increased. Taub and Raab also reported that the variability of $\mathrm{N}_{1}$ at a given signal level decreased as the background noise increased. The discrepancy in results may be due to basic differences in the recording and stimulus conditions. The earlier studies investigated the action potential of the eighth nerve in anesthetized animals, whereas we have investigated the EP at auditory centers more centrally located in unanesthetized animals. More importantly, whereas we worked within a range of signal-to-noise ratios of about $15 \mathrm{~dB}$ approximately centered around differential thresholds (75\% correct), the earlier studies covered a signal-to-noise ratio of up to $40 \mathrm{~dB}$ and did not attend closely to events near the differential thresholds.

Monaural vs Binaural Listening

The data reported so far have been for binaural stimulation, except where noted. Figure 10 compares thresholds obtained with the decision model at the inferior colliculus for ipsilateral, contralateral, and binaural signals. Here a small but reliable difference in detection performance can be seen with the contralateral signal at least 2 or $3 \mathrm{~dB}$ more detectable than either binaural or ipsilateral signals at threshold. ${ }^{7}$ One would not expect such differences from the human psychophysical literature, which predicts that binaural detection should be at least as good as monaural (Blodgett, Jeffress, \& Taylor, 1958).

These differences in threshold between monaural and binaural

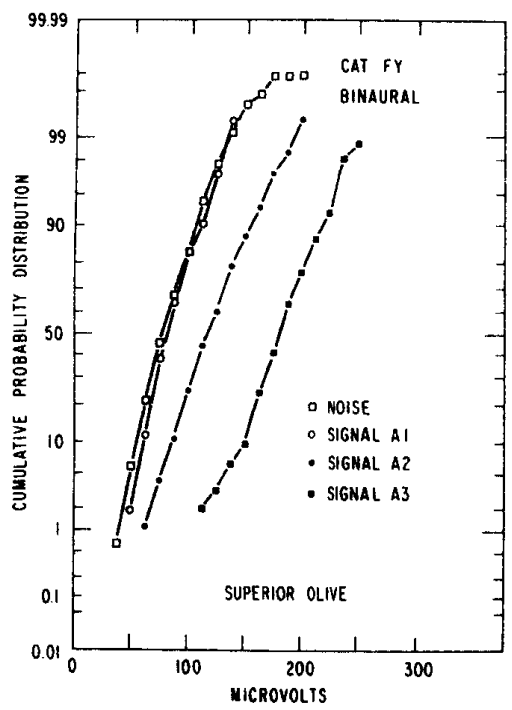

Fig. 6. Cumulative probability distributions of individual EP peak-to-peak amplitudes in microvolts plotted on normal probability paper. The distributions are identical to those plotted in Fig. 5 .

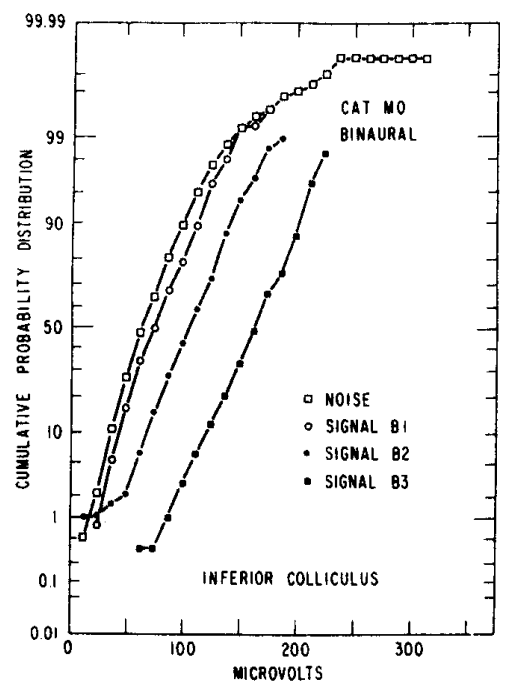

stimulation may be reflecting differences in the anatomical projection of each ear to a given inferior colliculus. Hind et al (1963) found that nerve cells driven by either ear usually responded at a higher spike rate to contralateral stimulation, that units excited by one ear and not the other were usually excited by the contralateral ear, and that binaural stimulation usually yielded lower spike counts than did monaural stimulation.

Below the inferior colliculus, at the superior olive binaural thresholds were as good or better than the monaural as one would expect (Fig. 11). Our superior olive placements were in the medial (accessory) nucleus, where interaural interactions have been found at both the EP and the unit level. These interactions, however, are varied and complex (Galambos et al, 1959; Hall, 1964; Moushegian, Rupert, \& Whitcomb, 1964), and no interpretation of the EP data in terms of them is attempted here. At the cochlear nucleus, only ipsilateral signals produced detectable physiological responses (i.e., above chance detection performance) for the range of signal energies investigated in this study. The addition of a contralateral signal did not affect this ipsilateral threshold.

\section{SUMMARY AND CONCLUSION}

In summary, when a simple decision rule is applied to the physiological sensory statistic of the amplitude of the evoked response measured at various auditory centers in the cat: (1) the decision model psychometric functions are similar to those of humans in the same detection task; 


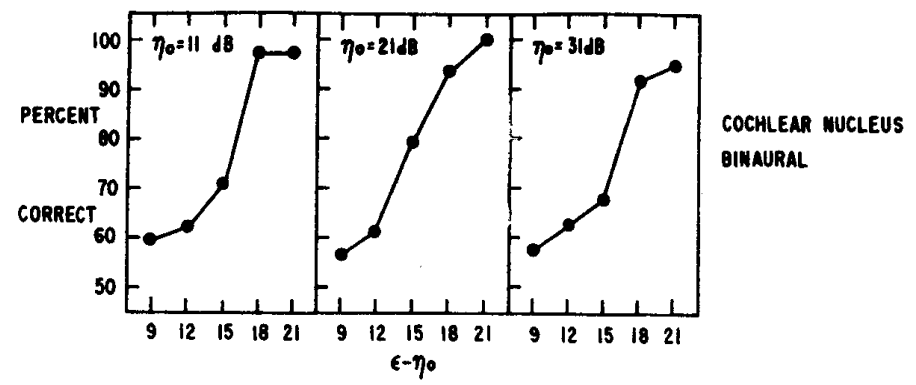

important to keep in mind that the only amplitude statistic to change with increased signal-to-noise ratio is the mean amplitude on signal trials; the mean amplitude on noise trials and the standard deviation on both signal trials and noise trials remained constant. If these amplitude changes were due simply to an increase in the number of units activated by the signal, we would expect the variance of the response amplitude to be proportional to its mean value. Although such a change makes sense statistically, it does not seem to describe what was found in our experiments. Presumably, instead, increasing the external noise levels may have its effect by increasing the number of units that are being driven at a saturated rate and by evoking responses in higher threshold units. As the noise level increases, we might assume that the size of the neuron pool available to respond to the signal increment is constant. So long as the number of units responding to the transient increase in external stimulation remained approximately the same for different background noise levels, we would expect the response-amplitude variance at threshold to be independent of the background noise.

Such an analysis suggests that a part of the change in the amplitude measure may be due to a change in the synchronization of the underlying impulse flow, rather than in the size of the population of units that are responding to the signal. The decreases in mean latency of the peak response and in the standard deviation of the latency with the signal level increase further support the idea that changes in the synchronization of the neural

(2) the decision model detection performance appears to obey Weber's law; (3) the mean amplitude of the EP increases systematically with signal level, but its variability is independent of both the signal energy and the background noise level being delivered to the system; (4) both the mean latency for the peaks of the EP and the variability of the latency decrease systematically with increased signal-to-noise ratios, suggesting that part of the change in the amplitude of the sensory response may be due to an increased synchronization in the underlying impulse flow; (5) the amplitude distributions are statistically nonnormal and tend to be skewed; however, they appear to be approximately normal over a lange extent of their range; and (6) interaural effects on the amplitude are found at the inferior colliculus, to a lesser degree at the superior olive, and not at all at the cochlear nucleus, possibly reflecting the characteristic innervation patterns of the single units in these different nuclei.

When considering how single-cell activity might give rise to the observed pattern of detection performance, it is

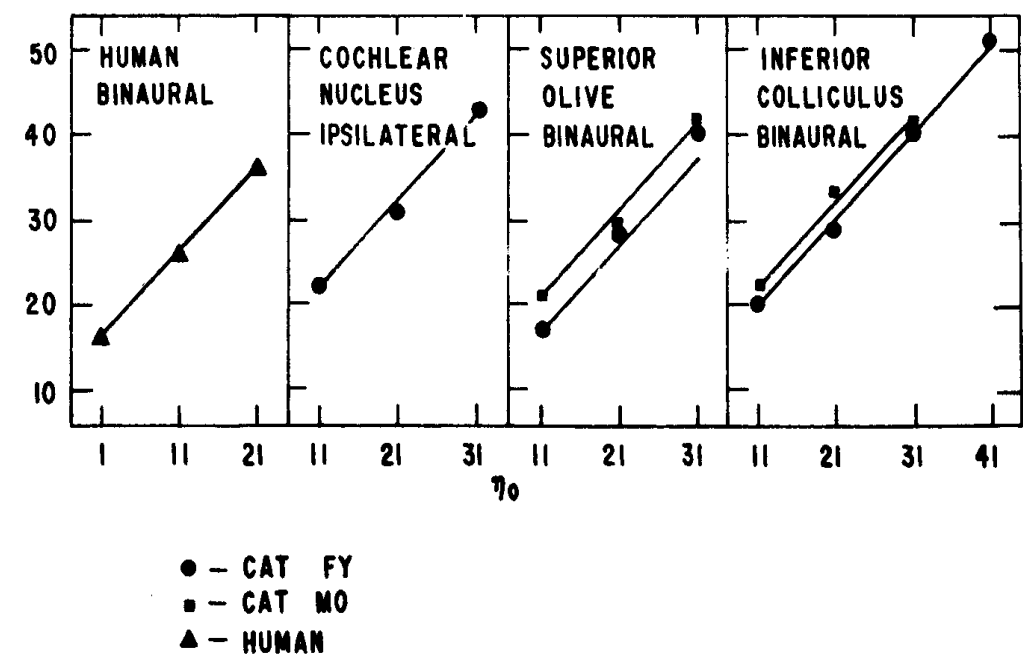

Fig. 8. Signal levels for $75 \%$ correct detection for the human and the decision model as a function of noise level. The points are interpolations from the linear segment of the psychometric functions. The solid lines are the prediction of Weber's law and have a slope of one. 
CAT FY

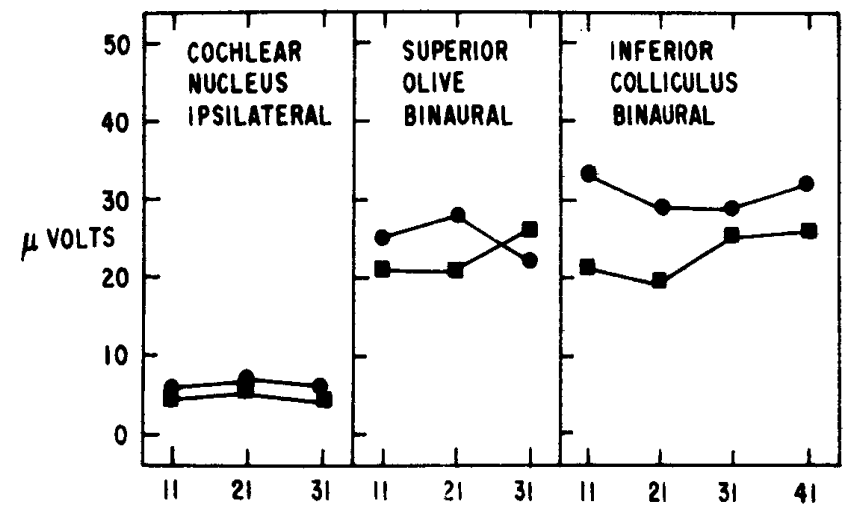

CAT MO

\section{- SIGNAL TRIALS \\ - NOISE tRIALS \\ STANOARD DEVIATION OF AMPLITUDE}

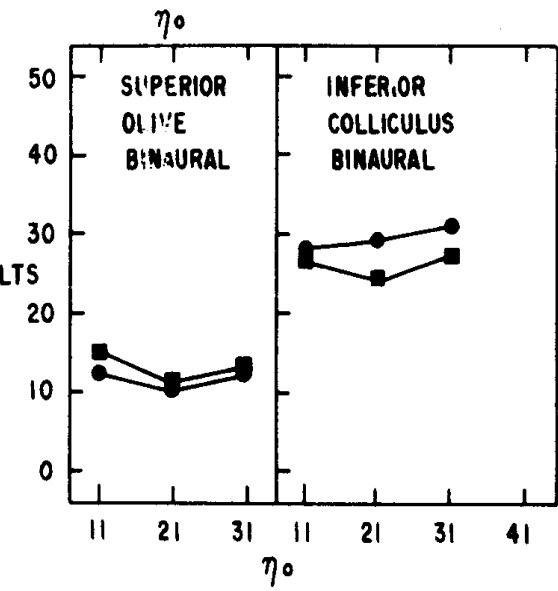

Fig. 9. The standard deviation of the EP amplitude at $\epsilon-\eta_{0}$ values generating 75\% correct detection performance at different $\eta_{0}$ levels. The data points were taken from $\epsilon-\eta_{0}$ ratios that generated the detection performance closest to 75\% correct for a given $\eta_{0}$ level. Each point represents approximately 50 trials. conditions on the EP statistics in any sensory system. Alterations in the external input can be converted directly into a change in the behavior of any specified decision model that uses the neural information to make decisions. This methodology may provide a useful bridge in investigating how an observed behavioral response is related to the neural events evoked by external signals.

\section{REFERENCES}

BLODGETT, H. C JEFFRESS, L, A. TAYLOR, R. W. Relation of matked threshold to sienal duration for various interaural phase-combinations. American Journal of Pyycholozy, 1958, 71 . 283-290.

FRISHKOPF, L. A. A probability approach to certain neuroelectric phenomena. M.I.T. Research Laboratory of Eiectronics Technical Report No. 307, 1 March, 1966.

GALAMBOS, R. SCHWARTZKOPFT, J., \& RUPERT, A. Microelectrode study of RUPERT, olivary nuclel. American Joumal of Physiology, 1959, 197, 527-536.

GREEN, D. M., MeKEY. M. J., \& LICKLIDER, J. C. R. Detection of a pulsed sinusoid in noise as a function of frequency. Joumal of the Acoustical Society of America, 1959, 29, 523-531. GREEN, D. M., SWETS, J. A. Signal detection theory and psychophysics, New Yort: Wiley, 1966. Pp. 78-81, 226-229.

HALL, J. L.. II. Binaural interaction in the accessory superior ollvary nucleus of the cat-An electrophysiological study of single neurons. M.I.T. Research Laboratory of Electronics Technical Report No. 416, 22 January, 1964. activity is an important sensory neural event underlying detection performance.

In general, the amplitude of the auditory evoked potential appears to provide very stable and sensitive information about the occurrence of external signals. When the amplitude measure is used as the decision statistic in a simple decision model, the detection performance of the model is consistent with human detection performance. The similarity between the performance of the two detection systems, the human $\mathbf{O}$ and the decision model, is an important prerequisite to serious consideration of any combination of decision model and neural statistic as being representative of the decision processes described in the theory of signal detection. It is, however, premature to implicate the evoked response amplitude in any brainstem nucleus as "the sensory statistic." The technique as elaborated here does provide the basis for the necessary investigations. These would include the trial-by-trial comparison of the performance of this and other decision models with that of the behaving animal.

This methodology can be applied to study the effects of changing signal

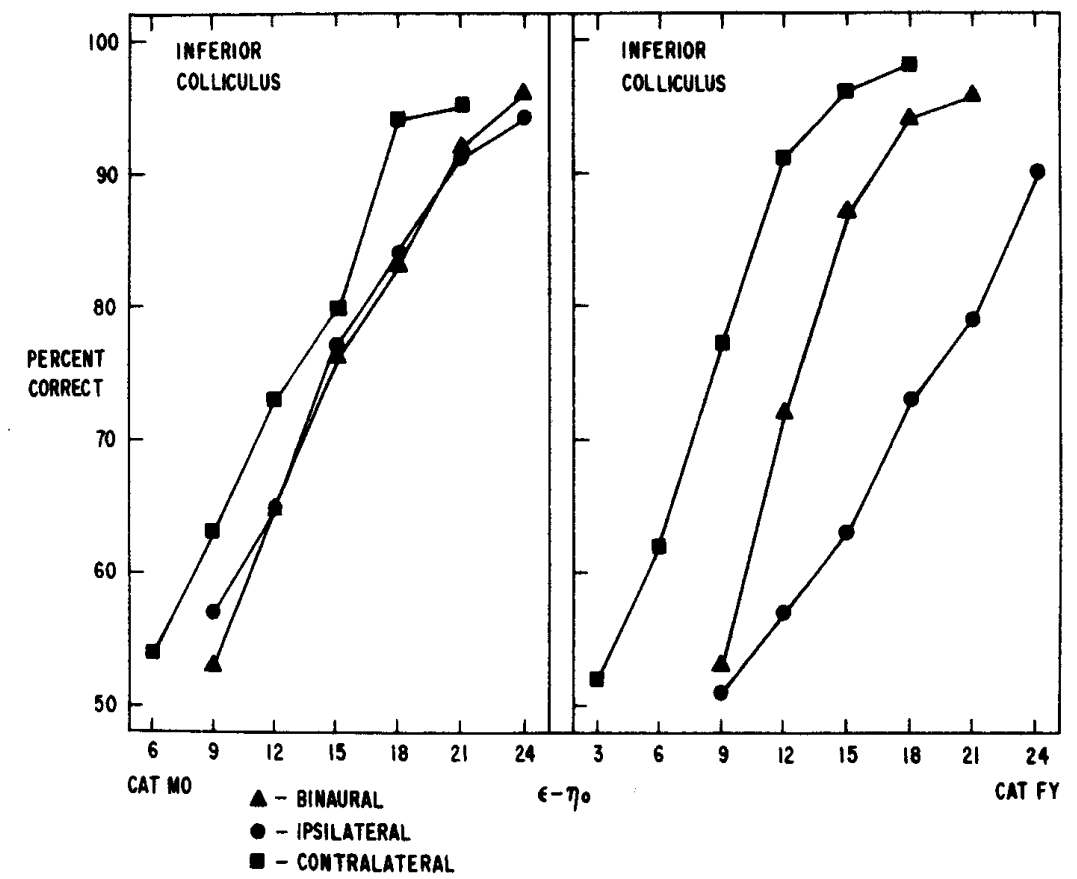

Fig. 10. Decision model psychometric functions generated by binaural, ipsilateral, and contralateral stimulation obtained from the inferior colliculus of both cats. The data points from Cat MO are based on 300 trials and three different noise levels. The data points from Cat FY are based on 400 trials and four different noise levels. 


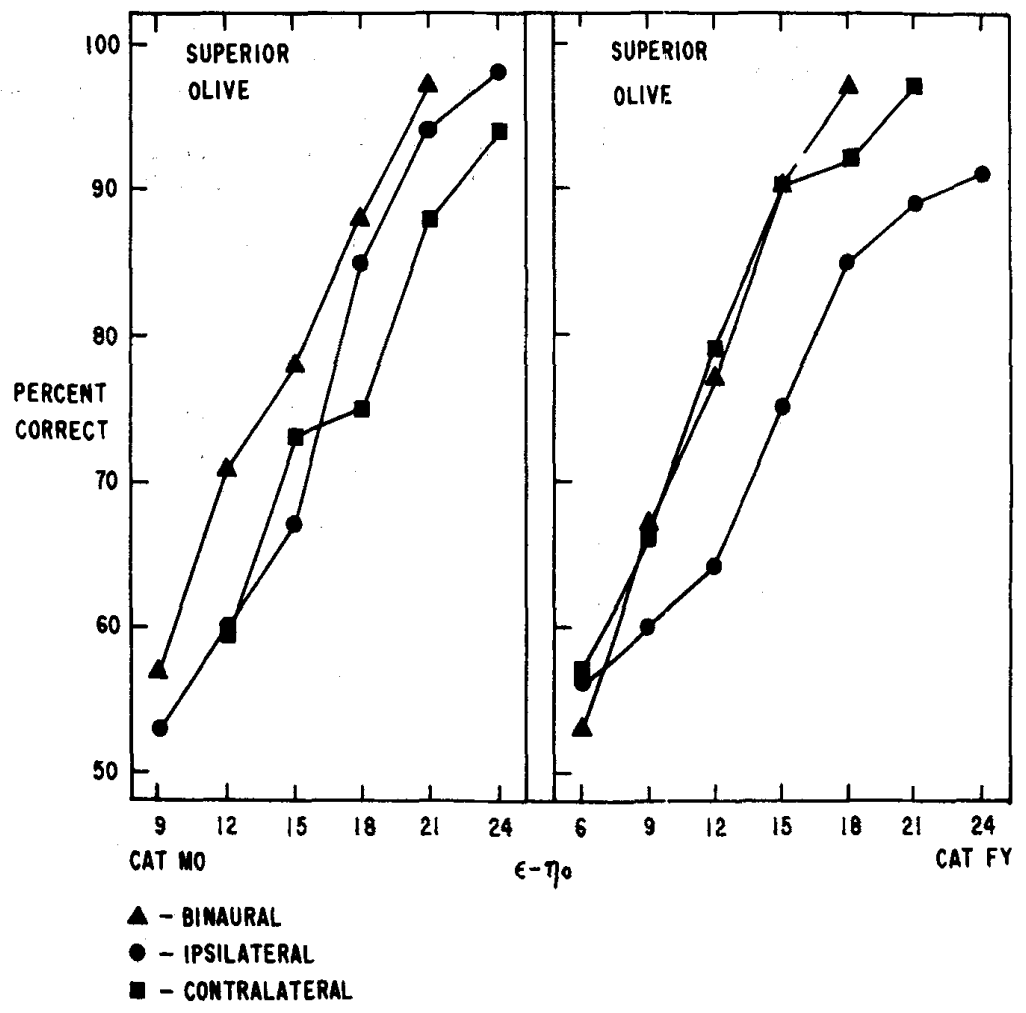

Fig. 11. Decision model psychometric functions generated by binaural, ipsilateral, and contralateral stimulation obtained from the superior olive of both cats. All data points are based on $\mathbf{3 0 0}$ trials and three different noise levels.

HILLY ARD, S. A., SQUIRES, K. C., BAUER, J. W., \& LINDSAY, P. H. Evoked potential correlates of auditory signal detection. Seience, 1971,172 , $1357-1360$.

HIN D, J. E., GOLDBERG, J. M., GREENWOOD, B. D., \& ROSE, J. E. Some discharge characteristics of single neurons in the inferior colliculus of the cat. II. Timing of the discharges and observations on binaural stimulation. Journal of Neurophysiology, 1963, 26, 321-341.

JOHN, E. R., \& MORGADES, P. P. Neural correlates of conditioned responses studied with multiple chronically
46, 969-978.

1. We included the older animal in the experiment in order to test the long-term stability of the results.

2. Signal-to-noise ratio is expressed in all figures in terms of 10 times the log of the signal energy, $\epsilon$, and 10 times the log of the noise power density, $\eta_{0}$. That is to say, $\epsilon-\eta_{0}=10 \log \mathrm{E} / \mathrm{N}_{0}$

3. Naturally, the amplitude and area statistics are correlated. Because the amplitude statistic appears to be slightly more sensitive than the area statistic for detection purposes, only the data obtained with the amplitude statistic are presented.

4. We use the standard terminology to designate the four possible stimulus-response combinations in a detection task: hit means the $S$ (i.e., the decision model) correctly detects a signal; miss, fails to detect a signal; false alarm, incorrectly reports a signal present; and correct rejection, correctly reports the absence of a signal.

5. Over all blocks of trials, the human $O$ 's criterion value ranged from a $\beta$ value of 0.99 to 1.04. $\beta$ is equal to the ratio of the probability densities of noise and signal-plus-noise distribution functions at the criterion point.

6. That the "psychometric functions" derived from the decision model are shallower than those from man may, in part, be due to the fact that the computer continued to make sporadic detection errors even at high signal-to-noise ratios. In the initial experiments, we expected this, since the decision model started with no information at all and the first few trials required guessing. Even with the inclusion of practice trials to establish an initial criterion, however, sporadic misses and false alarms still seemed to occur. It is possible that some of these errors were due to the movements and vocalizations of the cat contaminating the physiological recordings.

7. The detection performance obtained from the inferior colliculus stimulated ipsilaterally in Cat FY is poor compared to detection performance obtained with the other stimulus conditions. Threshold values obtained at other centers in Cat FY when this same ear was stimulated monaurally were also higher than those obtained from were also higher than those obtained from
the same nuclei in the other stimulus conditions. This suggests that the poor performance seen in the inferior colliculus ipsilaterally stimulated is due to anomalies in the peripheral mechanism of the ear. round window recordings to check out this possibility.

(Accepted for publication December I, 1971.) 